\title{
Non-setting, injectable biomaterials containing particulate hydroxyapatite can increase primary stability of bone screws in cancellous bone
}

\author{
Jorge Solana Muñoz ${ }^{\mathrm{a}}$, Ulrike Kettenberger ${ }^{\mathrm{a}}$, Philip Procter ${ }^{\mathrm{b}}$, Dominique P. Pioletti ${ }^{\mathrm{a}, *}$

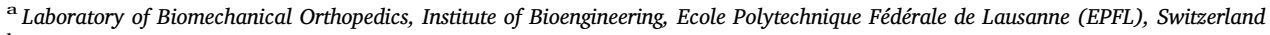 \\ ${ }^{\mathrm{b}}$ Applied Materials Science, Department of Engineering Sciences, Uppsala University, Sweden
}

\section{A R T I C L E I N F O}

\section{Keywords:}

Weak bone quality

Screw augmentation

Soft material

Hydrogel

Primary stability

Osteoporosis

\begin{abstract}
A B S T R A C T
Background: Fracture fixation in weak bone is still a clinical challenge. Screw augmentation was shown to successfully increase their primary stability. The currently used calcium phosphate or polymeric bone cements, however, present important drawbacks such as induced toxicity and/or impaired bone neo-formation. A new approach to enhance bone screw primary stability without affecting bone formation is the use of non-setting, calcium phosphate loaded soft materials as the augmentation material.

Methods: Two types of biomaterials (non-crosslinked hyaluronic acid as viscous fluid and agar as hydrogel) were loaded with $40 \mathrm{wt} / \mathrm{vol} \%$ of hydroxyapatite particles and characterized. The screw augmentation effect of all materials was evaluated through pull-out tests in bovine cancellous bone and compared to the non-augmented situation (control). The bone mineral density of each test sample was measured with $\mu$ CT scans and was used to normalize the pull-out strength.

Findings: Both materials loaded with hydroxyapatite increased the normalized pull-out strength of the screws compared to control samples and particle-free materials. This counter-intuitive augmentation effect increased with decreasing bone mineral density and was independent from the type of the soft materials used.

Interpretation: We were able to demonstrate that non-setting, injectable biomaterials loaded with ceramic particles can significantly enhance the primary stability of bone screws. This material combination opens the unique possibility to achieve a screw augmentation effect without impairing or even potentially favoring the bone formation in proximity to the screw. This effect would be particularly advantageous for the treatment of osteoporotic bone fractures requiring a stabilization with bone screws.
\end{abstract}

\section{Introduction}

Osteoporosis related fractures in the elderly population are an increasing burden on healthcare systems (Johnell and Kanis, 2006). Hip fracture is by far the most common reason for hospital admission, it may occurs either as a result of a fragility fracture (Acevedo et al., 2018) or a fall. Most hip fracture patients receive operative treatment (Weycker et al., 2016; Williamson et al., 2017). The ability of the proximal femur to bear the internal load actions resulting from a fall is significantly reduced by osteoporosis and in particular, it has been demonstrated that this is related to reduced areal bone mineral density (Curtis et al., 2016).

Each form of treatment following a bone fracture may result in undesired outcomes or complications. For example, in the use of screws to fixate undisplaced femoral neck fractures, the holding power of the screw may be limited by the degree of osteoporosis reduction in bone density in the regions where the surgeon tries to obtain purchase (Konstantinidis et al., 2016). Poor screw purchase may result in screw loosening and/or migration during the healing period and may necessitate a further operative intervention which is undesirable in elderly patients (Do et al., 2016; Rogmark et al., 2009). Differing clinical opinions on how to deal with this particular complication has led some surgeons to advocate going directly to an endoprosthesis or total hip replacement, whilst others have considered changing the design of the implant and/or modifying the surgical technique (Gardner et al., 2004). In this latter group, the use of cements to augment the fixation of the screw in the bone has been considered. This strategy has been the subject of many preclinical animal studies in which both poly methyl methacrylate (PMMA) and calcium phosphate (CaP) based injectable cements have been proposed as solutions (Stankewich et al., 1996; Stoffel et al., 2008; von der Linden et al., 2006). The purpose of cement augmentation is to improve the holding power of the screw and reduce

\footnotetext{
* Corresponding author at: EPFL/STI/IBI/LBO, Station 9, 1015 Lausanne, Switzerland.

E-mail address: dominique.pioletti@epfl.ch (D.P. Pioletti).
} 
the risk of migration during the fracture healing phase. Whilst there is good biomechanical support for this concept in ex vivo and in vivo preclinical models, however, in patients there is very little evidence that clinical outcomes are positively influenced. In intra capsular femoral neck fractures, there are also very few prospective randomized studies on cement augmentation. Mattsson and Larsson (2003) showed improved stability of internally fixed femoral neck fractures at one week and less pronounced at six weeks after surgery. The same authors later concluded that $\mathrm{CaP}$ cement augmentation did not improve results after internal fixation of displaced femoral neck fractures in a randomized study of 118 patients (Mattsson and Larsson, 2006). The same group also reported that in particular the use of injectable $\mathrm{CaP}$ resulted in impaired blood supply in femoral neck fractures (Mattsson et al., 2005).

Several alternative strategies have then been proposed to augment fixation of screws in poor quality cancellous bone (Parker and Handoll, 2009). The use of hydroxyapatite (HaP) coatings has been suggested by a number of authors, and Moroni in particular claims it was beneficial when hip screws were coated and used in fixing hip fractures (Moroni et al., 2004). The number of patients however was insufficient to justify such a conclusion. An Italian group looked at HaP coated implants long term using Dexa to assess the local bone formation and concluded that there was a significant increase in local bone density (Pesce et al., 2014). Alternatively, the local delivery of bone modifying drugs has also been considered. The use of Zoledronic (Zol) acid for example through a screw surface has shown to be feasible and beneficial in both pre-clinical (Greiner et al., 2008; Kettenberger et al., 2015; Peter et al., 2006) and clinical studies (Abtahi et al., 2012). Whilst this has not lead to any significant use outside of experimental studies, a dental implant pilot study has reached 5-year follow-up in patients showing less bone resorption close to the Zol delivering implants (Abtahi et al., 2016). For the coatings mentioned above, the effects are observed at mid to long term and neither is able to assure primary stability at the time of surgical intervention.

The present study hypothesis was formulated based on an earlier study in which Zol was delivered from hydroxyapatite particles embedded in a hydrogel matrix into peri-implant bone in a rat femoral model (Kettenberger et al., 2015). In this study, the control group of hydrogel loaded only with hydroxyapatite particles showed an unexpected mineralization of the hydrogel that led to an in situ formation of an osteoconductive scaffold. This high biological activity of the material with an induction of the body's own mineralization processes and an integration of the forming mineralized granules into viable bone makes this material combination highly attractive for bone augmentation. On the long term, this material seems to overcome the inherent limitations of bone cement such as the not existing or very slow resorption, the risk of leakage into articulations, and a potential impairment of the blood supply leading to an avascular necrosis of certain bone regions. It appears that the only major disadvantage of a hydroxyapatite-loaded hydrogel is its soft, non-setting consistency that is not expected to improve the primary stability of implants.

If it can be shown that the material combines both mechanical and biological performance and an improvement of the primary implant stability can be obtained, then this would potentially be of great clinical interest. Accordingly, the hypothesis in the present study is that the addition of an increased particulate percentage to soft biomaterials can significantly enhance the primary stability of bone screws in cancellous bone, whilst still remaining injectable.

\section{Methods}

\subsection{Design of the study}

Bovine distal femurs were used as sources of cancellous bone samples, which were prepared in the form of cylindrical cores of $20 \mathrm{~mm}$ of diameter and 20 to $25 \mathrm{~mm}$ of length. A titanium cancellous bone screw (ref: 604026, Cancellous Screw, Stryker, Selzach, Switzerland) was inserted into a predrilled hole that was coincident with the axis of each cylindrical bone sample. The mechanical influence of different biomaterials on cancellous bone screw stability was tested. The method used to compare the mechanical properties of those biomaterials was to inject each material into the screw hole prior to screw insertion and subsequent pull-out testing. The data from the pull-out testing and a micro-computerized tomography $(\mu \mathrm{CT})$ analysis of the trabecular bone density were used to interpret the effect of the tested biomaterials.

\subsection{Biomaterial preparation}

The study included 5 experimental groups, 4 groups for which the predrilled screw-holes were filled with a biomaterial and one group for which the holes were left empty (Control). The biomaterials were the following: the hydrogel agar with a concentration of $2 \mathrm{wt} / \mathrm{vol} \%$ pure (Agar) or loaded with $40 \mathrm{wt} / \mathrm{vol} \%$ of HaP (AgarHaP) and the viscous fluid hyaluronic acid with a concentration of $3.5 \mathrm{wt} / \mathrm{vol} \%$ pure (Hyalectine) or loaded with $40 \mathrm{wt} / \mathrm{vol} \%$ of HaP (HyalectineHaP). All products are commercially available (hydroxyapatite particles: ref 04238, hydroxyapatite, Sigma-Aldrich, Buchs, Switzerland; agar: ref 05039, AgarAgar, Sigma-Aldrich, Buchs, Switzerland; hyaluronic acid: Hyalectine, $800 \mathrm{kD}$, Fidia farmaceutici, Italy).

The preparation of the hydrogel used for the group Agar started by dissolving $2 \mathrm{wt} / \mathrm{vol} \%$ of agar in phosphate buffered saline (PBS, prepared in-house, $\mathrm{pH}$ 7.4) at room temperature (RT). Subsequently the solution was heated above $90^{\circ} \mathrm{C}$ to activate the agar and afterwards cooled down to RT. For the AgarHaP group, $40 \mathrm{wt} / \mathrm{vol} \%$ of HaP were additionally added to the agar solution prior to the heating step.

For the Hyalectine group, a non-crosslinked hyaluronic acid was used as injectable biomaterial. For its preparation, $3.5 \mathrm{wt} / \mathrm{vol} \%$ of Hyalectine powder was dissolved in PBS under continuous stirring with a laboratory spatula. For the HylectineHaP group, $40 \mathrm{wt} / \mathrm{vol} \%$ of $\mathrm{HaP}$ were added to the Hyalectine powder previously to the dissolution in PBS.

The 4 biomaterials were filled into labelled syringes and stored at $4^{\circ} \mathrm{C}$ until use.

\subsection{Bone sample preparation}

The bovine bones were obtained from a local slaughterhouse at the day of animal slaughter. To get the 5 experimental groups, about 120 bovine samples were extracted from 13 adult bovine distal femurs (condyles and trochlea, position of the extraction site shown in Fig. 1). Those samples were sorted following the validation criteria explained below. At the end, 63 bone samples were found to be usable, giving a number between 11 and 15 samples per experimental group. As first preparation step, all soft tissues around the bovine distal femurs were removed before placing the samples at $-20^{\circ} \mathrm{C}$ in the freezer. In a second step, the bone cylinders were extracted using a crown drill mounted in a column drill machine (Bosch PBD 40, Leinfelden-Echterdingen, Germany), turning at $200 \mathrm{rpm}$ and a band saw (Rexon BS12RA, Taichung, Taiwan) at its slowest speed. The extracted samples were cylinder-shaped with a diameter of $20 \mathrm{~mm}$ and a length of not $<20 \mathrm{~mm}$. The cortical layer was retained and the surface of the cortical bone was cleaned from remaining soft tissue to reduce the variance in drilling and screw insertion depth. Immediately after extraction, the samples were placed individually in laboratory tubes (Falcon $^{\mathrm{TM}}$ Conical Centrifuge Tube, $50 \mathrm{ml}$, Corning Life Sciences B.V., Amsterdam, The Netherlands) and kept in the freezer at $-20^{\circ} \mathrm{C}$ until further processing.

\subsection{Bone sample characterization and selection}

Micro-computerized tomography scans ( $\mu \mathrm{CT}$ ) (Skyscan 1076, Bruker microCT, Kontich, Belgium) of all samples were performed to 

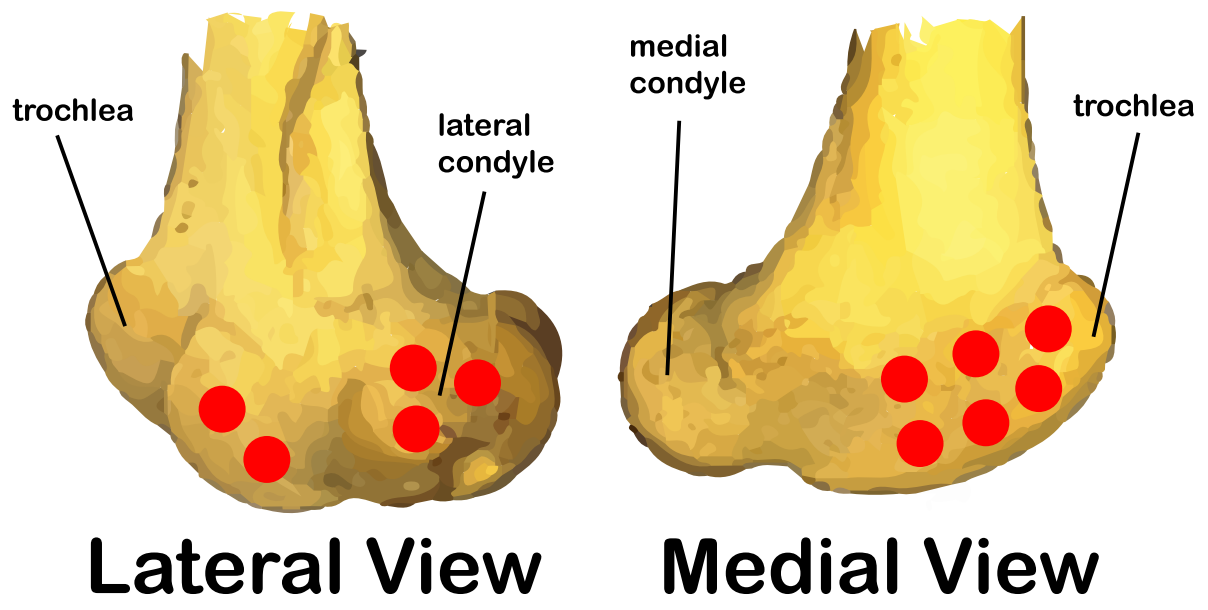

Fig. 1. Locations of bone sample extraction on bovine knee.
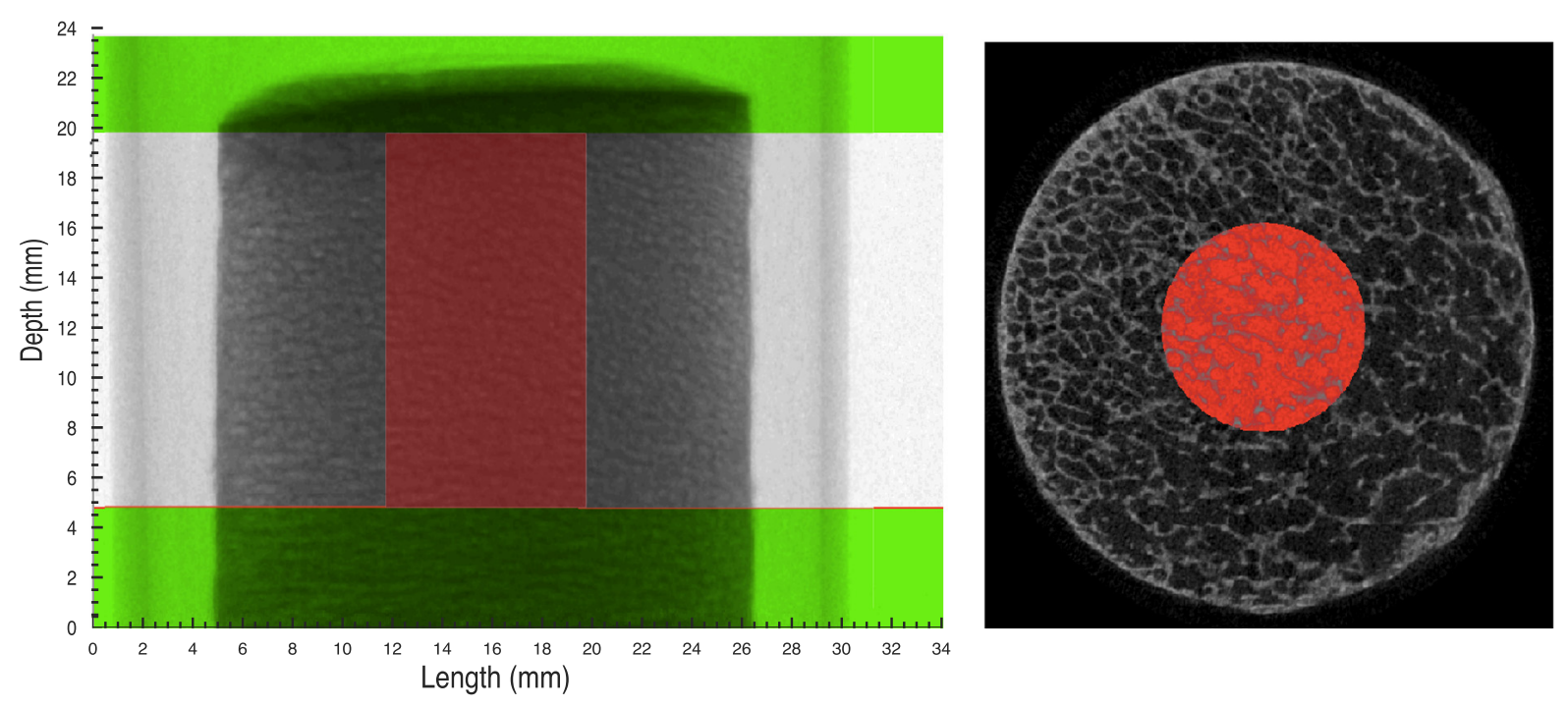

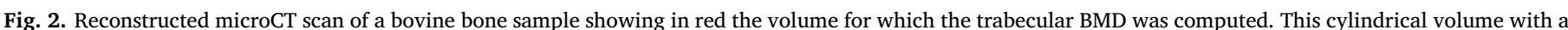

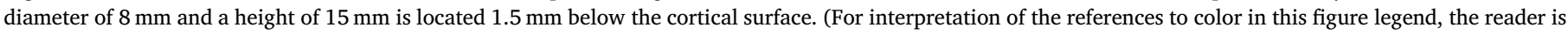
referred to the web version of this article.)

examine the bone quality before drilling the screw holes. The images were obtained using the following parameters: $1 \mathrm{~mm}$ aluminium filter, voltage $100 \mathrm{kV}$, current $100 \mu \mathrm{A}$, exposure time $120 \mathrm{~ms}$, rotation step $0.5^{\circ}$, angle coverage $180^{\circ}$ and spatial resolution $35 \mu \mathrm{m}$. A calibration of the attenuation coefficient versus bone mineral density (BMD in $\mathrm{g} / \mathrm{cm}^{3}$ ) was done for every session by scanning two cylindrical polymer-hydroxyapatite phantoms with a diameter of $16 \mathrm{~mm}$ and known mineral density under the same conditions than the bone samples. Image reconstruction was done with the software NRecon and GPURecon Server (Bruker microCT, Kontich, Belgium). The trabecular BMD values were obtained from a cylindrical region of about $8 \mathrm{~mm}$ of diameter and $15 \mathrm{~mm}$ of length at the centre of every bone sample located below the cortical shell (Fig. 2). This cylindrical region was defined large enough to include the bone region in which the cancellous screw (diameter $4 \mathrm{~mm}$ ) was anchored later on.

Three main aspects were analysed: the cancellous BMD, the cortical shell thickness, and the bone structure around the screw position. The BMD is generally used as part of the diagnosis protocol for osteopenia and osteoporosis (Cosman et al., 2014) and was correspondingly used as a normalization factor in this study. Every sample with a cortical shell thicker than $1.5 \mathrm{~mm}$ was rejected as, based on a previous study (Seebeck et al., 2005), it was demonstrated in human cadaver tibial bone that the pull-out strength is dominated by the cortical shell for a shell thicker than $1.5 \mathrm{~mm}$. Finally, a visual analysis of the reconstructed samples was used to exclude samples with atypical trabecular bone structure and samples that included part of the growth plate. To prevent the bone samples from drying, those were wrapped with standard laboratory plastic film (Parafilm M, Sigma-Aldrich, Steinheim, Germany) during the full scanning process and immediately placed back in the freezer at $-20{ }^{\circ} \mathrm{C}$ after the scan. During the scan, the frozen samples were exposed to room temperature and hence thawing for approximately $12 \mathrm{~min}$.

\subsection{Augmentation technique}

A similar procedure was followed for all the tested groups. In a first step, the samples were thawed inside the tubes by placing the tubes into a water bath with a temperature between $37^{\circ} \mathrm{C}$ and $40^{\circ} \mathrm{C}$. Then the column drill machine was used to drill holes with a diameter of $2.5 \mathrm{~mm}$ and a depth of $18 \mathrm{~mm}$, measured from the cortical surface, into the specimens with a standard drill bit. A $90^{\circ}$ angle between the drilling axis and the cortical surface was targeted. The screw hole depth was measured with the digital depth sensor of the drilling machine. Apart from the control group, between $0.1 \mathrm{ml}$ and $0.3 \mathrm{ml}$ of the different biomaterials (see Table 1 ) were injected into the screw holes with a positive displacement pipette (Distriman ${ }^{\circledR}$ with Distritip $^{\circledR} 1,25 \mathrm{ml}$, 
Table 1

The five tested groups with the hydrogels composition.

\begin{tabular}{lllll}
\hline Group & Agar & Hyalectine & HaP & Samples number \\
\hline Agar & $2 \%$ & - & - & 11 \\
AgarHaP & $2 \%$ & - & $40 \%$ & 13 \\
Hyalectine & - & $3.5 \%$ & - & 12 \\
HyalectineHaP & - & $3.5 \%$ & $40 \%$ & 12 \\
Control & - & - & - & 15 \\
\hline
\end{tabular}

Gilson, Middleton, USA) until an overflow was observed. The outlet diameter of this pipette tip is approximately $0.5 \mathrm{~mm}$ corresponding to the inner diameter of a $21 \mathrm{G}$ cannula. Commercially available orthopaedic cancellous bone screws with a length of $26 \mathrm{~mm}$ and a diameter of $4 \mathrm{~mm}$ (ref: 604026, Cancellous Screw, Stryker, Selzach, Switzerland) were manually inserted to a depth of $18 \mathrm{~mm}$ directly after biomaterial application. The correct insertion depth was controlled with a calliper. The measured screw head protrusion was $8 \pm 0.1 \mathrm{~mm}$.

\subsection{Mechanical pull-out tests procedure}

Before performing the pull-out test, the samples were fixed with a fast curing resin (Technovit 3040, Wehrheim, Germany) as proposed in earlier publications (Rincón-Kohli et al., 2009), into a specially designed mould that served equally as sample holder for the pull-out test. The mechanical tensile strength was applied by a tensile actuator (ref: E3000KZ832, Instron 3000, High Wycombe, England) at a rate of $5 \mathrm{~mm} / \mathrm{min}$, between the bone sample holder and the screw head holder. The data were acquired with an axial strength transducer (ref: 66901, Dynacell Dynamic Load Cell $5 \mathrm{kN}$, Instron) and automatically saved in an excel file. The pull-out strength was defined as the maximum tensile strength at the moment of bone failure (Fig. 3). The obtained pull-out strengths were normalized with the corresponding BMD of each bone sample. Linear curves were fitted to the results of each group to obtain the pull-out strength as function of the BMD. The relative differences in $\%$ between the resulting functions of the test groups and the function of the control group as reference were computed to obtain the augmentation effect of each tested biomaterial in relation to the BMD.

\subsection{Statistical analysis}

The software Matlab (MathWorks, Natick, United States) was used
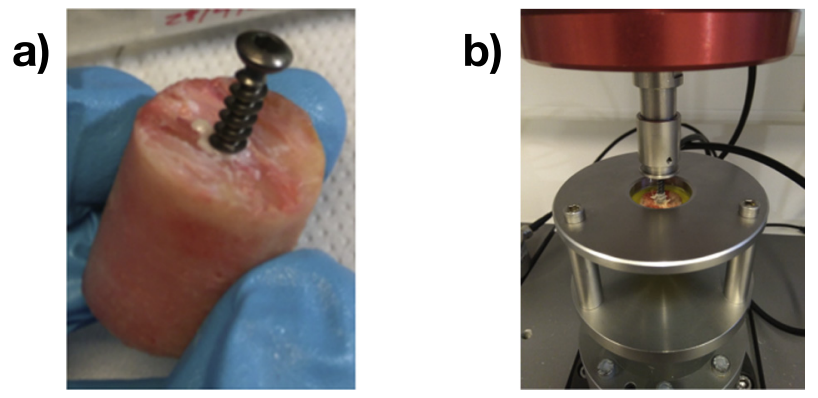

c)

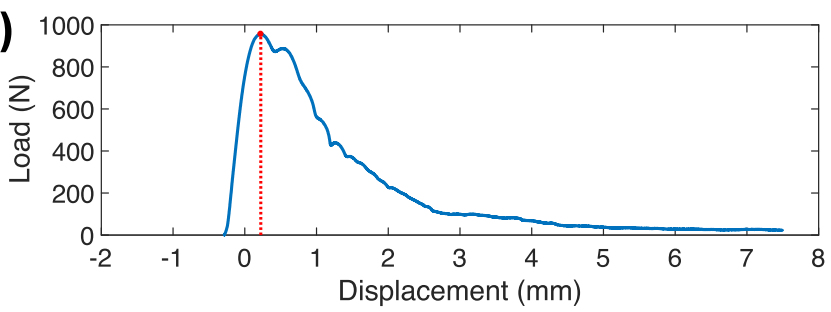

Fig. 3. a) Bone sample with an inserted screw; b) sample in the pull-out set-up; c) example of a typical pull-out test result. The pull-out strength was measured at the maximum of the curve (dotted line).
Table 2

Bovine mean and standard deviation (STD) of BMD $\left(\mathrm{g} / \mathrm{cm}^{3}\right)$ for each tested group.

\begin{tabular}{llll}
\hline \multirow{2}{*}{ Group } & \multicolumn{2}{l}{ BMD } & \\
\cline { 2 - 4 } & Mean & STD & Range \\
\hline Agar & 0.19 & 0.06 & 0.10 to 0.29 \\
AgarHaP & 0.20 & 0.09 & 0.09 to 0.35 \\
Hyalectine & 0.18 & 0.07 & 0.07 to 0.31 \\
HyalectineHaP & 0.21 & 0.08 & 0.11 to 0.33 \\
Control & 0.21 & 0.07 & 0.09 to 0.35 \\
\hline
\end{tabular}

for the statistical analysis of the results. A one-way ANOVA test followed by a multi-comparison test (Tuckey's honest significant difference criterion) was used to compare means of normalized pull-out strength between the different tested groups. A p value equal or lower than 0.05 was considered as significative.

\section{Results}

\subsection{Characterization of the bone samples}

The mean BMD and corresponding standard deviation for each group are reported in Table 2. No significant differences were observed between the BMD of the five experimental groups.

\subsection{Mechanical pull-out tests}

The normalized pull-out strengths of the five experimental groups are shown in Fig. 4. No significant differences were observed between the Control, the Hyalectine and the Agar groups indicating that a pure soft material does not have an effect on the primary stability of cancellous bone screws. However, significant differences were found between the two particle-loaded groups HyalectineHaP ( $p<0.0005)$ and AgarHaP ( $\mathrm{p}<0.001)$ and the Control. The results show an augmentation effect in the mean values of normalized pull-out strength of $27 \%$ for the group AgarHaP and $33 \%$ for HyalectineHaP when compared to the Control, which was $4481.4 \mathrm{~N} \mathrm{~cm}^{3} / \mathrm{g}$.

The computed augmentation effects of the AgarHaP and the HyalectineHaP group in relation to the BMD are shown in Fig. 5. The data show that the augmentation effect is strongest for a low BMD and decreases with increasing BMD values.

\section{Discussion}

To our knowledge, we were the first to hypothesize that a nonsetting soft material loaded with a sufficient amount of hydroxyapatite particles can improve the primary stability of cancellous bone screws. In this study, it was possible to confirm this hypothesis via a standard screw pull-out test with bovine cancellous bone samples. Two types of soft biomaterials, agar as hydrogel and hyaluronic acid as viscous fluid, were used pure or loaded with $40 \mathrm{wt} / \mathrm{vol} \%$ of $\mathrm{HaP}$ to fill the pre-drilled holes before screw insertion. Unfilled holes served as control. The results showed that the pure materials did not have any effect on the primary stability of the screws, the HaP loaded materials, however, significantly increased the pull-out strength. This augmentation effect was independent from the type of soft material used and increased with decreasing BMD.

At first glance, this positive effect of $\mathrm{HaP}$ loaded biomaterials on implant primary stability seems to be counter-intuitive as it is not expected that a non-setting, rather soft material can influence the pull-out strength. However, when looking at the literature, this finding is still aligned with earlier published discoveries. Steeves et al. found that the pull-out strength of cancellous bone screws increases with decreasing pilot hole diameter and explained this augmentation effect through the 


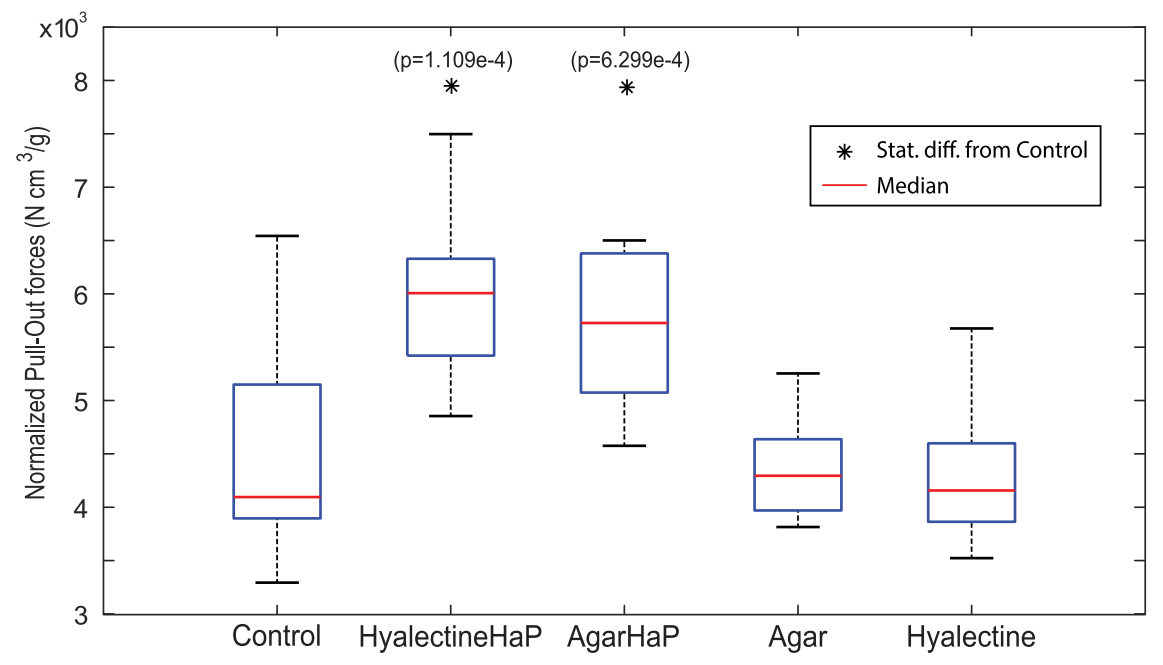

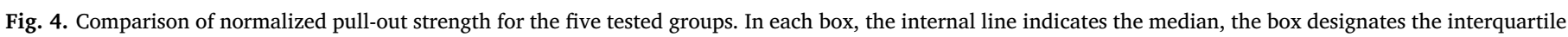

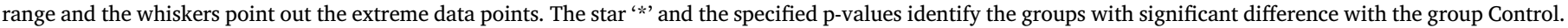

filling of the space between trabeculae with loose bone debris (Steeves et al., 2005). This effect was also described earlier by Öktenoğlu et al. (2001). As the HaP loaded materials of the here presented study are also pushed and compacted into the inter-trabecular space during the screw insertion, an accumulation of the hydroxyapatite between the trabeculae could be expected and cause the observed augmentation effect. Tami et al. applied hydroxyapatite particles alone in the pre-drilled screw holes in the proximal tibial metaphysis of OVX rats (Tami et al., 2009). Pull-out tests, histology, and microCT showed that the particles induced a stronger peri-implant bone mantle that improved the pull-out strength of the screws on the long term. However, no influence of the $\mathrm{HaP}$ on the primary stability of the screws was observed. This might be explained by the very small implant size in the rat model and the limited amount of HaP that could be applied to the pilot hole.

The challenge of obtaining adequate primary fixation of osteoporotic fractures has lead researchers and clinicians to consider the use of cements to augment fixation screws. Whilst the preclinical literature is broadly supportive of both calcium and PMMA based cements there is, so far, a lack of clinical support for the hoped-for benefits in hip fracture fixation. Accordingly, the results of the present study must be treated with caution until both the effectiveness and the biological safety have been validated in an appropriate preclinical animal model. It should be mentioned that the future clinical use of calcium-based cement in combination with screws to augment fixation of fractured neck of femur should probably be limited to the undisplaced grades where the risk of avascular necrosis is known to be less (Popelka et al., 2015).

The originality of the present study is to combine HaP and a soft, non-setting biomaterial. The aqueous, soft matrix makes the material injectable through a small needle and would significantly facilitate the application in clinics. Depending on the type of biomaterial used, the matrix itself can have further benefits. Hyaluronic acid, for example, is known to support healing processes and to be bacteriostatic and antiinflammatory (Carlson et al., 2004; Dahiya and Kamal, 2013).

The combination between hydroxyapatite and hyaluronic acid has also been shown to induce a rapid in vivo mineralization leading to the in situ formation of an osteoconductive scaffold when applied into poor quality, peri-implant bone (Kettenberger et al., 2015). However, it has to be noted that this study was performed with a much lower HaP content than the present study. A similar positive biological effect with a HaP content of $40 \mathrm{wt} / \mathrm{vol} \%$ in soft materials remains to be verified in an in vivo study.

There are some inherent limitations in the present study. First, the two soft materials used for this study, agar and non-crosslinked hyaluronic acid, only served as model substances and were chosen because of their simple preparation and handling. Agar is a hydrogel with a 3-

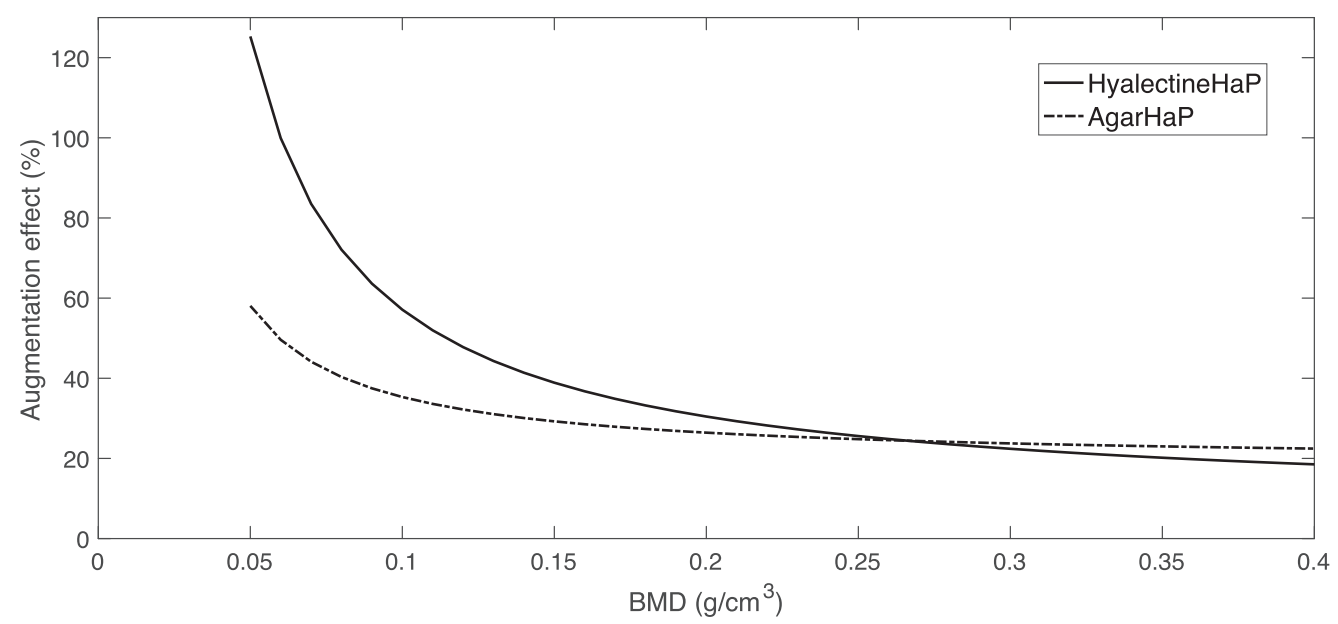

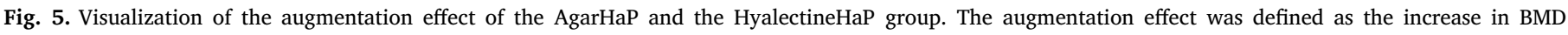
normalized pull-out strength compared to the Control group, within the assumption of linear correlation between pull-out strength and BMD. 
dimensional molecular network that is crushed into pieces when it is deformed, for example during injection. Non-crosslinked hyaluronic acid, in contrast, is a viscous fluid which is freely deformable due to a lacking polymer chain network. Non-crosslinked hyaluronic acid is characterized by a very high turnover in the human body and would be resorbed in vivo within several days (Stern, 2004). For an in vivo application, it is debatable if a non-cross-linked, quickly resorbable material is sufficient as transport medium for the hydroxyapatite particles or if a longer persisting, cross-linked matrix would be needed. It is unclear, if and how a rapid disappearance of the matrix influences the implant stability and the biological response over time. The presented experiments can only capture the situation immediately after screw insertion and animal tests would be needed for an evaluation of the temporal development of the implant integration and stability.

Another limitation of the presented study is the bovine origin of the bone. It is well known that the BMD of bovine bone is generally higher than the human ones. To partially circumvent this limitation, only the lowest BMD values of the bovine bone samples used for this study (mean BMD: $0.2021 \mathrm{~g} / \mathrm{cm}^{3}$ ) were within the range of values obtained from human femurs (neck mean BMD: $0.0888 \mathrm{~g} / \mathrm{cm}^{3}$, head mean BMD $0.1273 \mathrm{~g} / \mathrm{cm}^{3}$ ) reported in a recent study (Maeda et al., 2011). To decrease the importance of the BMD in the pull-out test, a normalization of the obtained pull-out strength by the BMD was proposed in the present study. From the mechanical point of view, whilst it is evident that there are differences in bone properties between human and the range of animal models in current use (Aerssens et al., 1998), there is some support for comparable properties (An, 1999). It has been demonstrated that there is reasonable equivalence between compressive strength and modulus of calf and human femoral neck bone (Ciarallo et al., 2006). Additionally, a study (Fletcher et al., 2018) concludes that juvenile bovine is a reasonable surrogate for human bone in screw pullout testing.

The conservation methods could have influenced the mechanical properties of the bone sample. Indeed, the bone samples were subjected to only one thaw-freeze cycle, which is typical to most biomechanical studies. Moreover, as all samples were processed in a similar way, even if a bias would have been induced by the conservation method, relative comparison of the mechanical tests between samples would have cancelled this bias. Due to technical problems during the experiments, some samples had to be withdrawn from the study leading to an uneven sample size between the groups. Finally for the limitation aspects of this study, the insertion of the screw may produce particulate bone that may tend to increase the insertion torque and consequently the pullout force (Ab-Lazid et al., 2014). However it is an assumption that this effect would be similar for both test and control groups and so the differences reported in Fig. 5 reflect the calcium particulate content.

The already observed biological advantages of HaP-loaded hydrogels and the augmentation effect on the primary stability of bone screws shown in this study make this composite biomaterial highly promising for fracture fixation in osteopenic or even osteoporotic bone. This material could overcome the biological limitations of current augmentation materials such as poor resorbability or possibly the risk of avascular necrosis with no or only little loss in the mechanical stabilization effect. The good injection properties of the proposed material makes it also suitable for minimal invasive application techniques. Further research, especially with suitable in vivo models, will be necessary to confirm the anticipated potential of this biomaterial.

\section{Acknowledgments}

This study was partially supported by BNF National Qualification Program (\# 2081_3). The authors gratefully acknowledge the donation of the Hyalectine materials used in this study by Fidia farmaceutici SpA.

\section{Conflict of interest}

None of the authors has any conflict of interest.

\section{References}

Ab-Lazid, R., Perilli, E., Ryan, M.K., Costi, J.J., Reynolds, K.J., 2014. Pullout strength of cancellous screws in human femoral heads depends on applied insertion torque, trabecular bone microarchitecture and areal bone mineral density. J. Mech. Behav. Biomed. Mater. 40, 354-361.

Abtahi, J., Tengvall, P., Aspenberg, P., 2012. A bisphosphonate-coating improves the fixation of metal implants in human bone. A randomized trial of dental implants. Bone 50, 1148-1151.

Abtahi, J., Henefalk, G., Aspenberg, P., 2016. Randomised trial of bisphosphonate-coated dental implants: radiographic follow-up after five years of loading. Int. J. Oral Maxillofac. Surg. 45, 1564-1569.

Acevedo, C., Stadelmann, V.A., Pioletti, D.P., Alliston, T., Ritchie, R.O., 2018. Fatigue as the missing link between bone fragility and fracture. Nat. Biomed. Eng. 2, 62-71.

Aerssens, J., Boonen, S., Lowet, G., Dequeker, J., 1998. Interspecies differences in bone composition, density, and quality: potential implications for in vivo bone research. Endocrinology 139, 663-670.

Carlson, G.A., Dragoo, J.L., Samimi, B., Bruckner, D.A., Bernard, G.W., Hedrick, M., Benhaim, P., 2004. Bacteriostatic properties of biomatrices against common orthopaedic pathogens. Biochem. Biophys. Res. Commun. 321, 472-478.

Ciarallo, A., Barralet, J., Tanzer, M., Kremer, R., 2006. An approach to compare the quality of cancellous bone from the femoral necks of healthy and osteoporotic patients through compression testing and microcomputed tomography imaging. Mcgill J. Med. 9, 102-107.

Cosman, F., Beur, S.J., Leboff, M.S., Lewiecki, E.M., Tanner, B., Randall, S., Lindsay, R. 2014. Clinician's Guide to Prevention and Treatment of Osteoporosis. Osteoporos. Int. 25, 2359-2381.

Curtis, E., Harvey, N., D'angelo, S., Cooper, C., Ward, K., Taylor, P., Pearson, G., Cooper, C., 2016. Bone mineral content and areal density, but not bone area, predict an incident fracture risk: a comparative study in a UK prospective cohort. Arch. Osteoporos. 11, 39.

Dahiya, P., Kamal, R., 2013. Hyaluronic acid: a boon in periodontal therapy. North Am. J. Med. Sci. 5, 309-315.

von der Linden, P., Gisep, A., Boner, V., Windolf, M., Appelt, A., Suhm, N., 2006. Biomechanical evaluation of a new augmentation method for enhanced screw fixation in osteoporotic proximal femoral fractures. J. Orthop. Res. 24, 2230-2237.

Do, L.N.D., Kruke, T.M., Foss, O.A., Basso, T., 2016. Reoperations and mortality in 383 patients operated with parallel screws for Garden I-II femoral neck fractures with up to ten years follow-up. Injury 47, 2739-2742.

Fletcher, J.W.A., Williams, S., Whitehouse, M.R., Gill, H.S., Preatoni, E., 2018. Juvenile bovine bone is an appropriate surrogate for normal and reduced density human bone in biomechanical testing: a validation study. Sci. Rep. 5, 10181.

Gardner, M.J., Lorich, D.G., Lane, J.M., 2004. Osteoporotic femoral neck fractures: management and current controversies. Instr. Course Lect. 53, 427-439.

Greiner, S.H., Wildemann, B., Back, D.A., Alidoust, M., Schwabe, P., Haas, N.P., Schmidmaier, G., 2008. Local application of zoledronic acid incorporated in a poly (D,L-lactide)-coated implant accelerates fracture healing in rats. Acta Orthop. 79, $717-725$.

Johnell, O., Kanis, J.A., 2006. An estimate of the worldwide prevalence and disability associated with osteoporotic fractures. Osteoporos. Int. 17, 1726-1733.

Kettenberger, U., Luginbuehl, V., Procter, P., Pioletti, D.P., 2017. In vitro and in vivo investigation of bisphosphonate-loaded hydroxyapatite particles for peri-implant bone augmentation. J. Tissue Eng. Regen. Med. 11, 1974-1985.

Konstantinidis, L., Helwig, P., Hirschmüller, A., Langenmair, E., Südkamp, N.P., Augat, P., 2016. When is the stability of a fracture fixation limited by osteoporotic bone? Injury 47, S27-S32.

Maeda, Y., Sugano, N., Saito, M., Yonenobu, K., 2011. Comparison of femoral morphology and bone mineral density between femoral neck fractures and trochanteric fractures. Clin. Orthop. Relat. Res. 469, 884-889.

Mattsson, P., Larsson, S., 2006. Calcium phosphate cement for augmentation did not improve results after internal fixation of displaced femoral neck fractures a randomized study of 118 patients. Acta Orthop. 77, 251-256.

Mattsson, P., Larsson, S., 2003. Stability of internally fixed femoral neck fractures augmented with resorbable cement A prospective randomized study using radiostereometry. Scand. J. Surg. 92, 215-219.

Mattsson, P., Alberts, A., Dahlberg, G., Sohlman, M., Hyldahl, H.C., Larsson, S., 2005. Resorbable cement for the augmentation of internally-fixed unstable trochanteric fractures a prospective, randomised multicentre study. J. Bone Joint Surg. (Br.) 8787, 1203-1209.

An, Y.H., An, Y.H., Draughn, R.A., 1999. Mechanical properties of bone. In: Mechanical Testing of Bone and the Bone-implant Interface. CRC Press, Boca Raton.

Moroni, A., Faldini, C., Pegreffi, F., Giannini, S., 2004. HA-coated screws decrease the incidence of fixation failure in osteoporotic trochanteric fractures. Clin. Orthop. 87-92 Aug.

Öktenoğlu, B.T., Ferrara, L.A., Andalkar, N., Özer, A.F., Sarioğlu, A.C., Benzel, E.C., 2001. Effects of hole preparation on screw pullout resistance and insertional torque: a biomechanical study. J. Neurosurg. 94, 91-96.

Parker, M.J., Handoll, H.H.G., 2009. Osteotomy, compression and other modifications of surgical techniques for internal fixation of extracapsular hip fractures. Cochrane Database Syst. Rev. 
Pesce, V., Maccagnano, G., Vicenti, G., Notarnicola, A., Moretti, L., Tafuri, S., Vanni, D., Salini, V., Moretti, B., 2014. The effect of hydroxyapatite coated screw in the lateral fragility fractures of the femur. A prospective randomized clinical study. J. Biol. Regul. Homeost. Agents 28, 125-132.

Peter, B., Gauthier, O., Laib, S., Bujoli, B., Guicheux, J., Janvier, P., van Lenthe, G.H., Muller, R., Zambelli, P.Y., Bouler, J.M., Pioletti, D.P., 2006. Local delivery of bisphosphonate from coated orthopedic implants increases implants mechanical stability in osteoporotic rats. J. Biomed. Mater. Res. 76, 133-143.

Popelka, O., Skála-Rosenbaum, J., Bartoška, R., Waldauf, P., Krbec, M., Džupa, V., 2015. Fracture type and injury-to-surgery interval as risk factors for avascular necrosis of the femoral head after internal fixation of intracapsular femoral neck fracture. Acta Chir. Orthop. Traumatol. Cechoslov. 82, 282-287.

Rincón-Kohli, L., Zysset, P.K., Rincón-Kohli, L., Zysset, P.K., 2009. Multi-axial mechanical properties of human trabecular bone. Biomech. Model. Mechanobiol. 8, 195-208.

Rogmark, C., Flensburg, L., Fredin, H., 2009. Undisplaced femoral neck fractures-no problems? A consecutive study of 224 patients treated with internal fixation. Injury 40, 274-276.

Seebeck, J., Goldhahn, J., Morlock, M.M., Schneider, E., 2005. Mechanical behavior of screws in normal and osteoporotic bone. Osteoporos. Int. 16, S107-S111.

Stankewich, C.J., Swiontkowski, M.F., Tencer, A.F., Yetkinler, D.N., Poser, R.D., 1996.
Augmentation of femoral neck fracture fixation with an injectable calcium-phosphate bone mineral cement. J. Orthop. Res. 14, 786-793.

Steeves, M., Stone, C., Mogaard, J., Byrne, S., 2005. How pilot-hole size affects bonescrew pullout strength in human cadaveric cancellous bone. Can. J. Surg. 48, 207-212.

Stern, R., 2004. Hyaluronan catabolism: a new metabolic pathway. Eur. J. Cell Biol. 83, $317-325$.

Stoffel, K.K., Leys, T., Damen, N., Nicholls, R.L., Kuster, M.S., 2008. A new technique for cement augmentation of the sliding hip screw in proximal femur fractures. Clin. Biomech. 23, 45-51.

Tami, A.E., Leitner, M.M., Baucke, M.G., Mueller, T.L., van Lenthe, G.H., Müller, R., Ito, K., 2009. Hydroxyapatite particles maintain peri-implant bone mantle during osseointegration in osteoporotic bone. Bone 45, 1117-1124.

Weycker, D., Li, X., Barron, R., Bornheimer, R., Chandler, D., 2016. Hospitalizations for Osteoporosis-related Fractures: economic Costs and Clinical Outcomes. Bone Report 5, 186-191.

Williamson, S., Landeiro, F., McConnell, T., Fulford-Smith, L., Javaid, M.K., Judge, A., Leal, J., 2017. Costs of fragility hip fractures globally: a systematic review and metaregression analysis. Osteoporos. Int. 28, 2791-2800. 